Article

\title{
Effect of Dietary Supplementation of Lactobacillus Casei YYL3 and L. Plantarum YYL5 on Growth, Immune Response and Intestinal Microbiota in Channel Catfish
}

\author{
Hongyu Zhang 1,2,3,4, Haibo Wang ${ }^{2,5}$, Kun Hu ${ }^{1,3,4}$, Liting Jiao ${ }^{2,5}$, Mingjun Zhao ${ }^{2}$, \\ Xianle Yang $1,3,4, *$ and Lei Xia ${ }^{2, *}$ \\ 1 National Pathogen Collection Center for Aquatic Animals, Shanghai Ocean University, Shanghai 210306, \\ China; zhhy02@126.com (H.Z.); khu@shou.edu.cn (K.H.) \\ 2 Chinese Academy of Fishery Sciences, Beijing 100141, China; xuehu1110@aliyun.com (H.W.); \\ jlttt666@163.com (L.J.); zhaomj@cafs.ac.cm (M.Z.) \\ 3 National Demonstration Center for Experimental Fisheries Science Education, Shanghai Ocean University, \\ Shanghai 210306, China \\ 4 Key Laboratory of Freshwater Aquatic Genetic Resources, Ministry of Agriculture, Shanghai 210306, China \\ 5 Beijing Seasun Aquaculture BIO TECH. Co.LTD, Beijing 102488, China \\ * Correspondence: xlyang@shou.edu.cn (X.Y.); xialei666@126.com (L.X.); \\ Tel.: +86-21-61900453 (X.Y.); +86-10-89360851 (L.X.)
}

Received: 10 October 2019; Accepted: 11 November 2019; Published: 20 November 2019

check for updates

Simple Summary: Channel catfish became one of the most efficient aquaculture species due to its fast growth and considerable commercial importance, however, the intensive farming of these fish resulted in disease pressure and the eutrophic conditions. In this study, we isolated two strains of lactobacillus, Lactobacillus casei YYL3 and L. plantarum YYL5, which can be potentially used as feed additives to promote the growth performance, disease resistance against Edwardsiella ictaluri, and dramatically change the composition of intestinal microbiota of channel catfish.

Abstract: The purpose of this study is to investigate the effect of probiotics L. casei YYL3 (Lc) and L. plantarum YYL5 (Lp) on growth performance, innate immunity, disease resistance and intestinal microbiota of channel catfish. A total of 252 catfish $(67.20 \pm 1.46 \mathrm{~g})$ were randomly divided into 3 groups which were fed with basal diet, Lc-added $\left(3.0 \times 10^{8} \mathrm{cfu} / \mathrm{g}\right)$ or Lp-added $\left(3.0 \times 10^{8} \mathrm{cfu} / \mathrm{g}\right)$ diets, respectively. After 4 weeks of feeding, Lc significantly enhanced the growth and feed utilization of channel catfish compared with the control group (CG). Following that, the catfish were challenged with an intraperitoneal injection of $200 \mu \mathrm{L}$ of the pathogenic E.ictaluri $\left(2.0 \times 10^{6} \mathrm{cfu} / \mathrm{mL}\right)$, the relative percent survival of Lc and Lp were $38.28 \%$ and $12.76 \%$, respectively. High-throughput sequencing indicated Lc and Lp reduced the alpha diversity of the intestinal microbiota in channel catfish. Lactobacillus were overwhelming in the guts during probiotics treatment, but almost vanished away after 2 weeks post-cessation of probiotics administration. Compared to CG, Lc and Lp resulted in an increased abundance of Pseudomonas and decreased amount of Aeromonas. Functional analysis revealed that Lc treatment upregulated the relative abundance of Kyoto Encyclopedia of Genes and Genomes (KEGG) pathways including lipid metabolism, metabolism of other amino acids, metabolism of terpenoids and polyketides, xenobiotics biodegradation and metabolism, and nucleotide metabolism. Combined, our data revealed that $\mathrm{Lc}$, as a feed additive at $3.0 \times 10^{8} \mathrm{cfu} / \mathrm{g}$, could promote the growth performance, disease resistance and dramatically change the composition of intestinal microbiota of channel catfish.

Keywords: Channel catfish; L. casei; L. plantarum; growth; disease resistance; intestinal microbiota 


\section{Introduction}

Channel Catfish is one of the top farm-raised fish in the United States and China with a total production of over several billion pounds per year [1,2]. Over the years, the emergence of infectious diseases caused by bacteria becomes the primary limiting factor in intensive aquaculture production of catfish in China. Especially, the diseases enteric septicemia and motile Aeromonas septicemia caused by Edwardsiella ictaluri and Aeromonas hydrophila respectively are responsible for significant economic impacts to the channel catfish industry throughout the world [3,4]. Thus, broad-spectrum chemotherapeutic agents such as antibiotics has been used to treat these diseases, however, excessive and imprudent use of antibiotics leads to the emergence of resistant bacteria strains and environment and food safety problems. To mitigate these problems, probiotics have been suggested to be an alternative way for the prevention and control of fish health associated problems $[5,6]$.

Lactic acid bacteria (LAB) have a reputation of being "generally regarded as safe" and are widely used in aquaculture $[7,8]$. L. plantarum is one of the widely used LAB in aquaculture and benefits to aquatic organisms by immunostimulation, disease resistance and growth enhancement [9-13]. For example, Son et al. [14] reported that treatment of a diet containing L. plantarum at the level of $10^{8} \mathrm{cfu} / \mathrm{g}$ for 4 weeks significantly increased percent weight gain and feed efficiency, and reduced their mortality rate more than $20 \%$ after challenged with Streptococcu ssp. Beck et al. [15] showed that L. plantarum feeding for 30 days increased survival rate of olive flounder which was challenged with E. tarda $\left(10^{5} \mathrm{cfu} / \mathrm{g}\right.$ fish) compared with control, probably through enhancing expression of proinflammatory genes (T-bet, IL-1 $\beta$, and IFN- $\gamma$ ) and CD18. In Giri's study [16], they found that administration of L. plantarum (VSG3) for 60 days significantly improved the growth, immunity and disease resistance of Labeo rohita.

Compared with L. plantarum, less attention was paid to L. casei, although it is also commonly used in aquaculture [17]. Lamari et al. [18] demonstrated that L. casei X2 improved the growth performance of sea bass larvae. Abasali and Mohamad [19] found that L. casei in combination with L. acidophilus, Enterococcus faecium, and Bifidobacterium thermophilum could increase the gonadosomatic index and the production of fingerlings Xiphophorus helleri at reproductive age.

We previously isolated Lc and Lp from commercial probiotics, which showed significant inhibitory activity against the main bacterial pathogens, i.e., E. ictaluri, A. hydrophila and Yersinia ruckeri, in channel catfish in vitro $[4,20]$ (data not published). However, their in vivo activities are unknown, especially, their effects on growth, immunity response, and intestinal bacteria of channel catfish remain elusive. Therefore, the present study was conducted to investigate and compare the efficacy of Lc and Lp on the growth performance, immunity, gut microflora and disease resistance against E. ictaluri in channel catfish.

\section{Materials and Methods}

\subsection{Ethics Statement}

This study was approved by the Animal Care and Use Committee of the Green Fish Drug Innovation Center at the Chinese Academy of Fishery Sciences.

\subsection{Probiotic Strains and Culture Conditions}

Lp and Lc tested in this study were isolated from commercial probiotics in Beijing Seasun Aquaculture BIO TECH. Co. LTD and identified based on the phenotypic characteristics and $16 \mathrm{~S}$ rRNA gene sequencing. They were grown at $37^{\circ} \mathrm{C}$ for $24 \mathrm{~h}$ in MRS broth (BaseBio, Hangzhou, China), and then spun down at $5000 \times \mathrm{g}$ for $5 \mathrm{~min}$. The pellets were washed once, and then resuspended in sterile $0.85 \% \mathrm{NaCl}$ solution. The number of the bacterial cells in the suspension was determined by MRS agar (BaseBio) plate count [12]. 


\subsection{Experimental Diets}

The basal diet was modified from the work of $\mathrm{Li}$ et al. [21]. The formulation and proximate analysis of the experimental diets are shown in Table 1 . The basal diet was used as control, and the other two diets were prepared by supplementing Lc $\left(3.0 \times 10^{8} \mathrm{cfu} / \mathrm{g}\right)$ and Lp $\left(3.0 \times 10^{8} \mathrm{cfu} / \mathrm{g}\right)$ respectively. Powdered dietary ingredients were thoroughly mixed and blended with oil, water, and appropriate amount of Lactobacillus until a soft dough formed. The dough was cut into particles with appropriate size through mincer, then the particles were dried down to a moisture content less than $8 \%$ in a drying cabinet using an air blower at $37^{\circ} \mathrm{C}$. The feed were prepared every week and stored in sealed plastic bags at $-20^{\circ} \mathrm{C}$ to maintain the viability of probiotic [22,23]. The viability of the bacterial cells was assessed by MRS agar (BaseBio) plate count. All experimental diets were formulated to be isonitrogenous and isocaloric.

Table 1. Composition and nutrient content of basal diet (expressed as \%).

\begin{tabular}{cc}
\hline Ingredient & Proportion \\
\hline Fish meal & 8.00 \\
Soybean meal & 45.00 \\
Corn meal & 25.00 \\
Wheat middling & 7.40 \\
Corn oil & 3.60 \\
Dicalcium phosphate & 1.00 \\
Vitamin mix 1 & 0.50 \\
Mineral mix ${ }^{2}$ & 0.50 \\
Cellulose & 6.00 \\
Carboxymethyl cellulose & 3.00 \\
Proximate composition & \\
Moisture & 7.84 \\
Protein & 30.56 \\
Lipid & 5.76 \\
Ash & 6.84
\end{tabular}

${ }^{1}$ Vitamin premix $\left(\mathrm{mg} / \mathrm{kg}\right.$ dry diet): $\mathrm{V}_{\mathrm{A}}, 8 ; \mathrm{V}_{\mathrm{D} 3}, 2 ; \mathrm{V}_{\mathrm{K}}, 10 ; \mathrm{V}_{\mathrm{E}}, 200 ; \mathrm{V}_{\mathrm{B} 1}, 10 ; \mathrm{V}_{\mathrm{B} 2}, 12 ; \mathrm{V}_{\mathrm{B} 6}, 10 ;$ calcium pantothenate,32; nicotinic acid, 80; folic acid, 2; $\mathrm{V}_{\mathrm{B} 12}, 0.01 ; \mathrm{V}_{\mathrm{H}}, 0.2$; choline chloride, $400 ; \mathrm{V}_{\mathrm{C}}, 400 .{ }^{2}$ Mineral premix (mg/kg dry diet): $\mathrm{ZnSO}_{4} \cdot 7 \mathrm{H}_{2} \mathrm{O}, 150 ; \mathrm{FeSO}_{4} \cdot 7 \mathrm{H}_{2} \mathrm{O}, 40 ; \mathrm{MnSO}_{4} \cdot 7 \mathrm{H}_{2} \mathrm{O}, 25 ; \mathrm{CuCl}_{2}, 3 ; \mathrm{KI}, 5 ; \mathrm{CoCl}_{2} \cdot 6 \mathrm{H}_{2} \mathrm{O}, 0.05 ; \mathrm{Na}_{2} \mathrm{SeO}_{3}, 0.09$.

\subsection{Fish and Rearing Conditions}

Channel catfish were obtained from Beijing Longchi Aquaculture Farm, Beijing, China, showing no signs of disease through gross and microscopic examination of skin, gills, and guts of representative samples. Upon arrival at laboratory, fish were stocked in four $3 \mathrm{~m}^{3}$ tanks and acclimatized with laboratory conditions for 2 weeks. Thereafter, 252 fish $(67.20 \pm 1.46 \mathrm{~g})$ were randomly distributed into 9 glass aquariums ( $300 \mathrm{~L}$ ) at density of 28 fish per tank and fed experimental diets for 4 weeks. During the feeding trial, fish were fed experimental diets twice per day (at 9:00 a.m. and 4:00 p.m.) up to apparent satiation. Any uneaten portion was collected after feeding and immediately dried in an oven at $80^{\circ} \mathrm{C}$. The amounts of all diets fed were calculated by subtracting the uneaten portions and recorded daily. During the culture period, one-third of the water was renewed every 2 days, and the water quality was maintained at the following range: water temperature $26.0-27.0{ }^{\circ} \mathrm{C}, \mathrm{pH} 7.2-7.8$ and dissolved oxygen $6.2-6.7 \mathrm{mg} / \mathrm{L}$. Fish in each aquarium were group weighted and counted at the end of the trail to determine weight gain (WG) and survival. WG, survival rate, specific growth rate (SGR) and feed conversion ratio (FCR) were calculated using formulae described by Gupta et al. [24].

$$
\begin{gathered}
W G(g)=\text { final weight }- \text { initial weight } \\
\text { Survival rate }(\%)=\left(\frac{\text { fish no. of } \text { fish }}{\text { initial no. of fish }}\right) \times 100 \%
\end{gathered}
$$




$$
\begin{gathered}
S G R(\%)=\left(\frac{\ln \text { final body weight }-\ln \text { initial body weight }}{\text { period of culture }(\text { days })}\right) \times 100 \% \\
F C R(\%)=\left(\frac{\text { total dry feed } f e d(g)}{\text { total live weight gain }(g)}\right) \times 100 \%
\end{gathered}
$$

\subsection{Non-Specific Immune Parameters of Serum}

After 4 weeks growth trial, 15 fish from each group were euthanized on ice for analysis of non-specific immune parameters of serum. Blood samples were collected through the caudal vein from 5 fish per tank using a $1 \mathrm{~mL}$ syringe at the end of the feeding trial, and immediately transferred the Eppendorf tubes without anticoagulant, after blood tubes have been allowed to clot $(1 \mathrm{~h}$ at room temperature and $4 \mathrm{~h}$ at $\left.4{ }^{\circ} \mathrm{C}\right)$ and centrifuged $\left(1500 \times \mathrm{g}, 5 \mathrm{~min}, 4^{\circ} \mathrm{C}\right)$, the serum was obtained and stored at $-20^{\circ} \mathrm{C}$ until assay [25].

Alternative complement pathway activity $\left(\mathrm{ACH}_{50}\right)$ was determined using the method described by Doan [25]:

$$
A C H_{50}(\text { units } / m l)=1 / K \times r \times 1 / 2
$$

Where $\mathrm{K}$ is the amount of serum giving $50 \%$ hemolysis, $\mathrm{r}$ is the reciprocal of the serum dilution, and $1 / 2$ is the correction factor. The assay was performed on a $1 / 2$ scale of the original method $[25,26]$.

Serum lysozyme (LZM) and Superoxide dismutase (SOD) activities were determined by LZM and SOD Assay Kits (Nanjing Jiancheng Bioengineering Institute, China) according to the manufacturer's instructions.

\subsection{Challenge Test}

E. ictaluri was kindly provided by Dr Zhou (Yangtze River Fisheries Research Institute, Chinese Academy of Fishery Sciences). The strain was incubated at $28^{\circ} \mathrm{C}, 150 \mathrm{rpm}$ for $24 \mathrm{~h}$ in nutrient broth media (BaseBio). The cultures were centrifuged at $5000 \times \mathrm{g}$ for $5 \mathrm{~min}$. The pellets were washed twice with sterile $0.85 \% \mathrm{NaCl}$ solution. The number of the bacterial cells in the suspensions was measured by nutrient agar (BaseBio) plate count. At the end of the feeding trail, 54 fish from each group (Lc, Lp and CG) were challenged intraperitoneally with $200 \mu \mathrm{l}\left(2.0 \times 10^{6} \mathrm{cfu} / \mathrm{mL}\right)$ of E. ictaluri, and then redistributed evenly to 3 glass tanks. The challenge test lasted for 15 days, and all fish were fed twice daily with basal diet. Fish mortality for each tank was recorded daily, and cumulative mortality and RPS were calculated by the following formula [27].

$$
\begin{gathered}
\text { Cumulative mortalatiy }(\%)=\left(\frac{\text { Total mortality in each treatment after challenge }}{\text { Total number of fish challenged for same treatment }}\right) \times 100 \\
\operatorname{RPS}(\%)=\left(1-\frac{\text { Percentage mortality in probiotic groups }}{\text { Percent mortality in control group }}\right) \times 100
\end{gathered}
$$

\subsection{Sample Collection and DNA Extraction}

The intestinal flora was sampled after 4 weeks (right after feeding trial) and 6 weeks ( 2 weeks extra after cessation of probiotics), respectively. Fish were fasted for $24 \mathrm{~h}$ before sampling. Three samples (one fish per replicate) randomly selected from each group at each time point, were anesthetized with eugenol, brain tissue was destroyed with anatomic needle. The abdomen of fish was cleaned with $70 \%$ ethanol, and the abdominal cavity was opened under aseptic condition. The whole intestine was taken out and $1 \mathrm{~cm}$ anterior and posterior intestine was discarded. The intestinal contents were extruded into a $1.5 \mathrm{~mL}$ sterile tube, then the whole intestinal tract was dissected, rinsed with sterile phosphate buffer saline (PBS) for three times, and centrifuged to collect the residual content.

Bacterial DNA was extracted using the E.Z.N.A Mag-Bind Soil DNA Kit (Omega, Norcross, GA, USA) according to the manufacturer's protocol, and Qubit ${ }^{2} 2.0$ (life, Carlsbad, CA, USA) was 
used to measure the concentration of the DNA. A total of 18 DNA samples were submitted to Sangon Biotech, Inc. (Shanghai, China) for PCR amplification and Next-Generation Sequencing using Illumina Miseq platform. The V3-V4 region of the bacteria $16 \mathrm{~S}$ ribosomal RNA gene from each sample were amplified using the bacterial universal primer 341F (5'-CCTACACGACG CTCTTCCGATCTG(barcode)CCTACGGGNGGCWGCAG-3') and 805R (5'-GACTGGAGTTCCTT GGCACCCGAGAATTCCAGACTACHVGGGTATCTAATCC-3'), where barcode is an six-base sequence unique to each sample. The PCR was performed as described previously [28]. AMPure $\mathrm{XP}$ beads was used to purify the free primers and primer dimer species in the amplicon product, and universal Illumina adaptor and index was used for library construction. Before sequencing, the DNA concentration of each PCR product was determined using a Qubit@2.0 Green double-stranded DNA assay and it was quality controlled using a bioanalyzer (Agilent 2100, Santa Clara, CA, USA). Depending on coverage needs, all libraries can be pooled for one run. The amplicons from each reaction mixture were pooled in equimolar ratios based on their concentration. Sequencing was performed using the Illumina MiSeq system (Illumina MiSeq, San Diego, CA, USA), according to the manufacturer's instructions.

\subsection{Bioinformatic Analysis}

The paired end sequence reads were quality trimmed using the Cutadapt tool (Version 1.2.1) to remove adaptors, barcodes, primer sequences. Paired end reads were merged using the PEAR tool (Version 0.9.6) [29], and low-quality reads ( $Q$ score $<20$ ) were removed using Prinseq (Version 0.20.4) [30]. After de-multiplexing and quality filtering of the raw sequence reads, reference-based and de novo chimeras were checked and removed from the cleaned sequences and operational taxonomic unit (OTU) clustering was performed with a 0.97 threshold using Usearch (Version 5.2.236) [31]. The analyses of alpha diversity indexes comprising community diversity (Simpson, Shannon index and Good's coverage) and richness (ACE and Chao-1), which were calculated using Mothur (Version 1.30.1) [32]. The beta diversity and taxon composition were analyzed using QIIME (version 1.8) for calculating weighted UniFrac. Principal coordinates analysis (PCoA) was used to evaluate the Beta diversity obtained by weighted UniFrac analyses [33]. Weighted PCoA 3D figure was created using the vegan (version 2.0-10) in $\mathrm{R}$ (version 3.2), and heatmap of genus was generated using gplots (version 2.17.0) package in $\mathrm{R}$ (version 3.2).

To predict the functional profiles of microbial communities, the Phylogenetic Investigation of Communities by Reconstruction of Unobserved States (PICRUSt) method was used [34]. Briefly, 16S rRNA gene sequences were clustered into OTUs using the closed reference OTU picking algorithm and the Greengenes reference taxonomy (Greengenes 13.5). The predicted 16S copy number was used to normalize the OTU table for each representative sequence. Molecular functions for each sample were predicted by categorizing annotated metagenome sequences using KEGG Orthology database into KEGG pathways. A two-sided Welch's t-test was used to identify enriched metabolic pathways in the microbiota of catfish fed with probiotics by software STAMP, with $p<0.05$ considered significant.

\subsection{Statistical Analysis}

All data are expressed as mean \pm SD. The statistical significance between means of the independent groups was analyzed using one-way ANOVA followed by LSD (Least-Significant Difference), and $p$ value less than 0.05 was statistically significant.

\section{Results}

\subsection{Growth Performance}

Growth parameters at the end of 4 weeks feeding trail are presented in Table 2, the Lc has significant higher WG and SGR than those in the CG and Lp. Besides, the Lc showed significant lower 
FCR than the CG $(p<0.05)$. No significant difference in survival rate was observed among Lc, Lp, and CG $(p>0.05)$.

Table 2. Growth performance of channel catfish fed with experimental diets for 4 weeks (mean \pm SD).

\begin{tabular}{cccc}
\hline & CG & Lc & Lp \\
\hline Initial weight (g) & $67.43 \pm 1.31$ & $67.18 \pm 1.42$ & $67.01 \pm 1.64$ \\
Final weight (g) & $108.40 \pm 9.57^{\mathrm{b}}$ & $114.67 \pm 10.75^{\mathrm{a}}$ & $110.57 \pm 10.61^{\mathrm{b}}$ \\
WG (g) & $40.95 \pm 0.94^{\mathrm{b}}$ & $47.49 \pm 1.62^{\mathrm{a}}$ & $43.56 \pm 1.77^{\mathrm{b}}$ \\
Survival rate (\%) & $98.81 \pm 2.06^{\mathrm{a}}$ & $98.81 \pm 2.06^{\mathrm{a}}$ & $100.00 \pm 0.00^{\mathrm{a}}$ \\
SGR (\%) & $1.69 \pm 0.03^{\mathrm{b}}$ & $1.90 \pm 0.05^{\mathrm{a}}$ & $1.79 \pm 0.06^{\mathrm{b}}$ \\
FCR (\%) & $0.98 \pm 0.07^{\mathrm{a}}$ & $0.82 \pm 0.02^{\mathrm{b}}$ & $0.89 \pm 0.04^{\mathrm{ab}}$
\end{tabular}

$\mathrm{a}-\mathrm{b}$ In the same line, means with different letters are significantly different $(p<0.05)$, means with the same letters are not significantly different $(p>0.05)$. CG, basal diet; Lc, basal diet with $3.0 \times 10^{8} \mathrm{cfu} / \mathrm{g}$ of $L$. casei YYL3; Lp, basal diet with $3.0 \times 10^{8} \mathrm{cfu} / \mathrm{g}$ of L. plantanum YYL5.

\subsection{Immune Parameters}

After 4 weeks feeding trail, Lc significantly enhanced LZM activity than CG and Lp group $(p<0.05)$, but Lp has no effect on LZM compared with CG $(p>0.05)$ (Figure 1A). Lc and Lp didn't affect $\mathrm{ACH}_{50}$ and SOD compared with CG (Figure 1B,C).
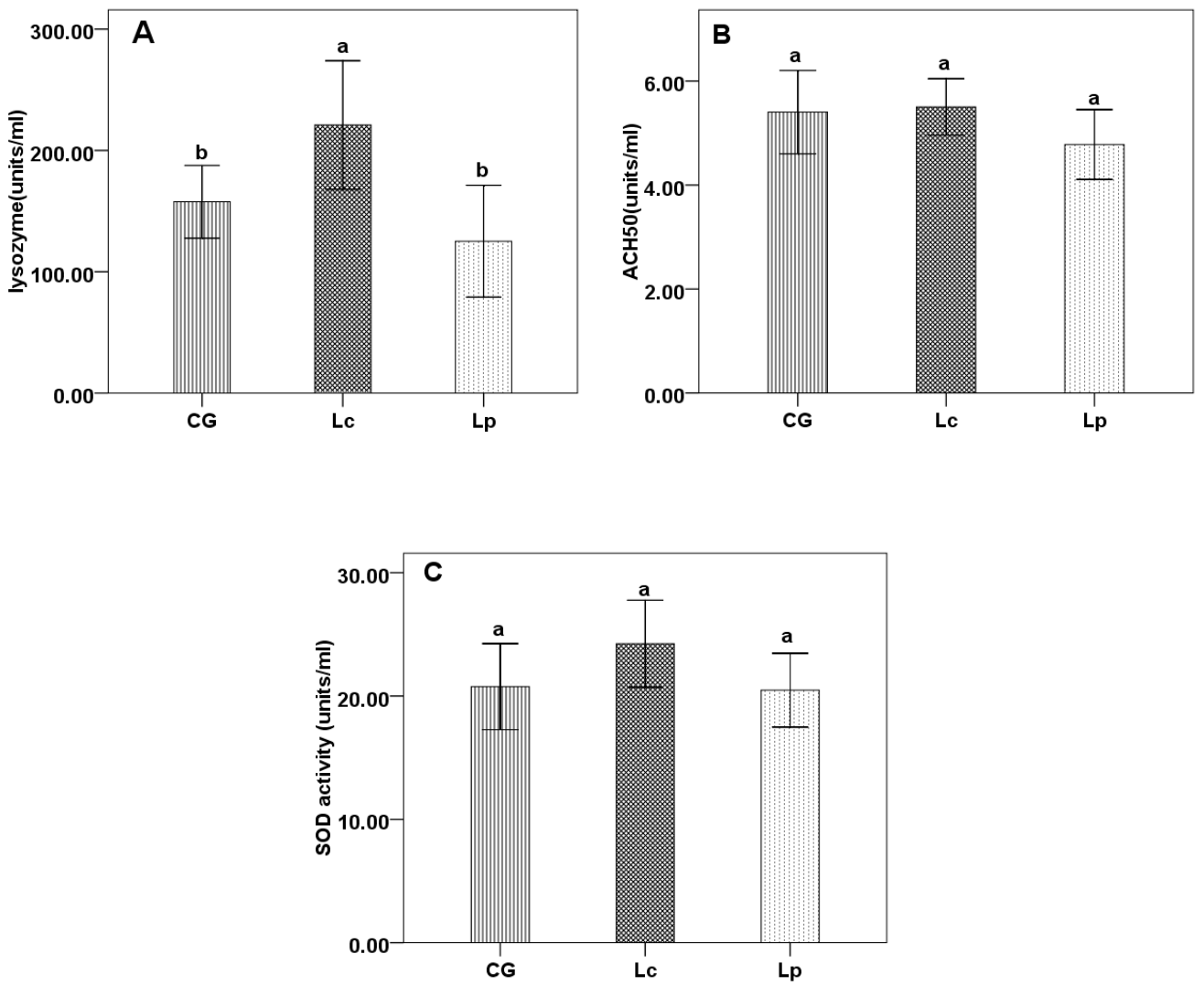

Figure 1. Lysozyme (A), alternative complement pathway activity $\left(\mathrm{ACH}_{50}\right)(\mathbf{B})$, and superoxide dismutase (SOD) (C) of channel catfish fed the three diets for 4 weeks. CG: basal diet; Lc: basal diet with L. casei YYL3; Lp: basal diet with L. plantanum YYL5. Each value represents mean \pm SD $(n=5)$. Different letters are significantly $(p<0.05)$ different by LSD test. 


\subsection{Challenge Test}

The challenge test showed that supplementation of Lc enhanced the protection against E. ictaluri infection. Lc $(53.70 \pm 8.49) \%$ had lower cumulative mortality than CG $(87.02 \pm 6.42) \%$ and Lp $(75.93 \pm$ $11.56) \%(p<0.05)$ (Figure 2). The RPS of Lc and Lp were $38.28 \%$ and $12.76 \%$, respectively.

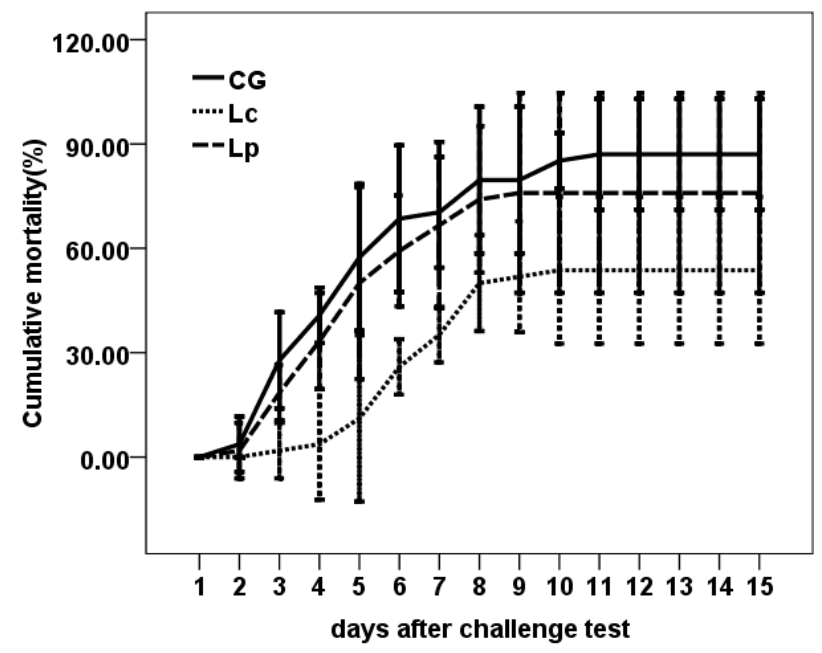

Figure 2. Cumulative mortality of channel catfish after challenged with the pathogen, E. ictaluri.

\subsection{Characteristics of the High-Throughput Sequence Data}

After quality filtration and adapter trimming of raw reads, a total of 1,024,202 valid sequences were obtained from all channel catfish intestinal microbiota samples. Then they are clustered into 8727 OTUs using a $97 \%$ sequence identity cutoff. To assess the sequencing depth and species richness, a rarefaction curve was constructed for each sample.

The rarefaction curves (Figure 3) indicated that the sufficient sampling depth was achieved for each sample.

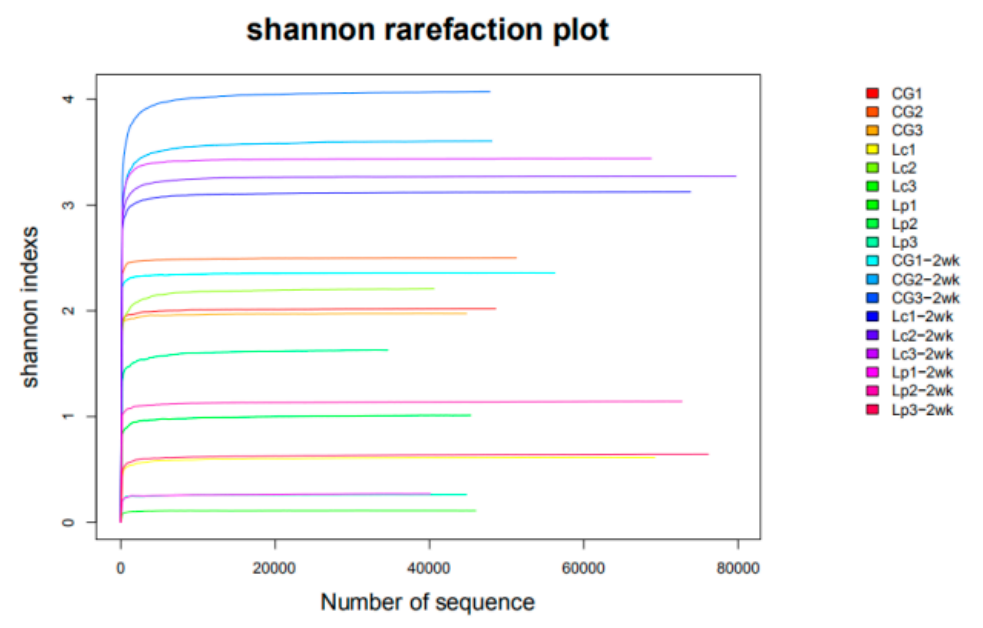

Figure 3. Shannon rarefaction curves of catfish intestinal microbiota samples which were harvest at 4 and 6 weeks. Note: CG: control group fed with basal diet for 4 weeks; Lc: fed with basal diet supplemented with $3.0 \times 10^{8} \mathrm{cfu} / \mathrm{g}$ of L. casei for 4 weeks; Lp: fed with basal diet supplemented with $3.0 \times 10^{8} \mathrm{cfu} / \mathrm{g}$ of L. plantarum for 4 weeks; CG-2wk: CG group continue to feed basal diet for two more weeks; Lc-2wk: Lc group feeding basal diet instead of the probiotics diet for two more weeks; Lp-2wk: Lp group feeding basal diet instead of the probiotics diet for two more weeks. The following is the same. 


\subsection{Diversity Analysis}

To compare the bacterial diversity across groups, bacterial richness and diversity indices were calculated from the proportion of OUTs (Table 3). The Good's coverage of each group was above 99\%, indicating an adequate depth of sequencing. Right after 4 weeks probiotics feeding trials, CG had significantly higher species richness estimated by ACE $(1041.25 \pm 199.74)$ than Lc $(472.49 \pm 316.03)$ and Lp (531.92 \pm 148.74$)$, and also higher bacterial diversity estimated by Simpson index $(0.21 \pm 0.06)$ than Lc $(0.75 \pm 0.28)$ and $\operatorname{Lp}(0.73 \pm 0.19)$. Two weeks feeding of basal diet after cessation of probiotic administration resulted in that the community diversity in Lp-2wk, but not Lc- $2 w k$, recovered to the levels of CG and CG-2wk as evidenced by ACE, Chao1 index, and Goods coverage.

Table 3. The intestinal microbiota diversity index of each group (mean $\pm \mathrm{SD}$ ).

\begin{tabular}{cccccc}
\hline Sample & Shannon Index & ACE & Chao1 Index & Goods Coverage & Simpson \\
\hline CG & $2.16 \pm 0.29^{\mathrm{ab}}$ & $1041.25 \pm 199.74^{\mathrm{a}}$ & $600.46 \pm 53.19^{\mathrm{ab}}$ & $0.997 \pm 0.000^{\mathrm{c}}$ & $0.21 \pm 0.06^{\mathrm{b}}$ \\
Lc & $0.98 \pm 1.10^{\mathrm{bc}}$ & $472.49 \pm 316.03^{\mathrm{b}}$ & $455.37 \pm 324.30^{\mathrm{b}}$ & $0.999 \pm 0.001^{\mathrm{a}}$ & $0.75 \pm 0.28^{\mathrm{a}}$ \\
Lp & $0.97 \pm 0.68^{\mathrm{bc}}$ & $531.92 \pm 148.74^{\mathrm{b}}$ & $466.16 \pm 192.22^{\mathrm{b}}$ & $0.998 \pm 0.001^{\mathrm{b}}$ & $0.73 \pm 0.19^{\mathrm{a}}$ \\
CG-2wk & $3.34 \pm 0.88^{\mathrm{a}}$ & $1048.15 \pm 358.86^{\mathrm{a}}$ & $1008.51 \pm 489.45^{\mathrm{a}}$ & $0.997 \pm 0.000^{\mathrm{bc}}$ & $0.16 \pm 0.03^{\mathrm{b}}$ \\
Lc-2wk & $3.28 \pm 0.16^{\mathrm{a}}$ & $513.82 \pm 30.60^{\mathrm{b}}$ & $518.77 \pm 22.11^{\mathrm{b}}$ & $0.999 \pm 0.000^{\mathrm{a}}$ & $0.10 \pm 0.01^{\mathrm{b}}$ \\
Lp-2wk & $0.69 \pm 0.44^{\mathrm{c}}$ & $808.84 \pm 60.62^{\mathrm{ab}}$ & $640.22 \pm 126.39^{\mathrm{ab}}$ & $0.997 \pm 0.000^{\mathrm{bc}}$ & $0.74 \pm 0.25^{\mathrm{a}}$ \\
\hline
\end{tabular}

Note: In the same column, different superscript letters denote significant difference $(p<0.05)$, while the same letters are not significantly different $(p>0.05)$.

A PCoA analysis of the weighted UniFrac distances (Figure 4) displayed that the samples were segregated by the type of diet, with the Lc and Lp samples clearly distinct from the CG samples right after 4 weeks probiotic feeding trials. Following 2 weeks of basal diet feeding after cessation of probiotic tests, Lp-2wk and CG-2wk grouped together, which were separated from Lc- 2 wk group, suggesting that the diversity of Lp-2wk was closer to CG-2wk than Lc-2wk. This was also supported by heatmap of genus results which showed that Lp-2wk, CG and CG-2wk were clustered together (Figure S1).

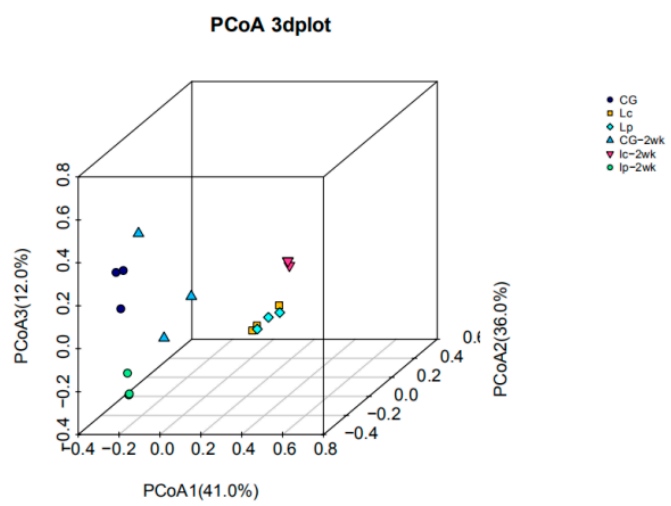

Figure 4. PCoA 3D figure of community compositions in catfish intestinal microbiota based on UniFrac distance matrix. CG, blue circle; Lc, yellow square; Lp, cyan diamond; CG-2wk, cyan triangle; Lc-2wk, purple triangle; Lp-2wk, green circle.

\subsection{Changes in Community Structure and Intestinal Microbiota Abundance in the Catfish}

To further investigate the changes of gut microbial communities after feeding of probiotics, bacterial phyla and genus frequencies from 3 groups of samples were analyzed (Figure 5A). We found CG samples have 3 major phyla, i.e., Fusobacteria (38.25\%), Bacteroidetes (29.37\%), and Firmicutes $(27.46 \%$ ), while Lp and Lc samples were dominated by Firmicutes (accounting for $>95 \%$ of $16 \mathrm{~S}$ reads). Two weeks after cessation of probiotic administration, the intestinal flora in CG did not change much, as evidenced by the top 5 abundant phyla (Fusobacteria, Bacteroidetes, Firmicutes, Proteobacteria and 
Verrucomicrobia) (Table S1). While the average number of Firmicutes in Lc-2wk and Lp-2wk dropped sharply from $>95 \%$ to $6.69 \%$ and $0.53 \%$, respectively. Their dominant phyla become Proteobacteria $(81.4 \%)$ and Fusobacteria $(84.72 \%)$, respectively.
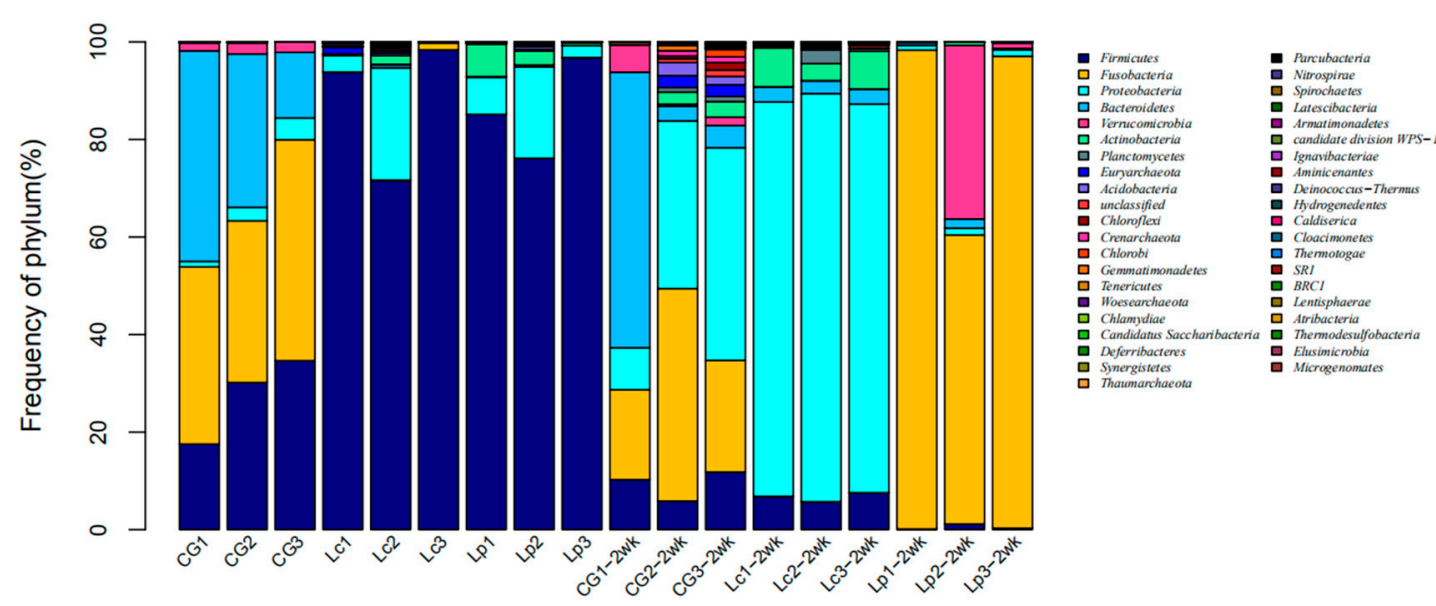

(A)

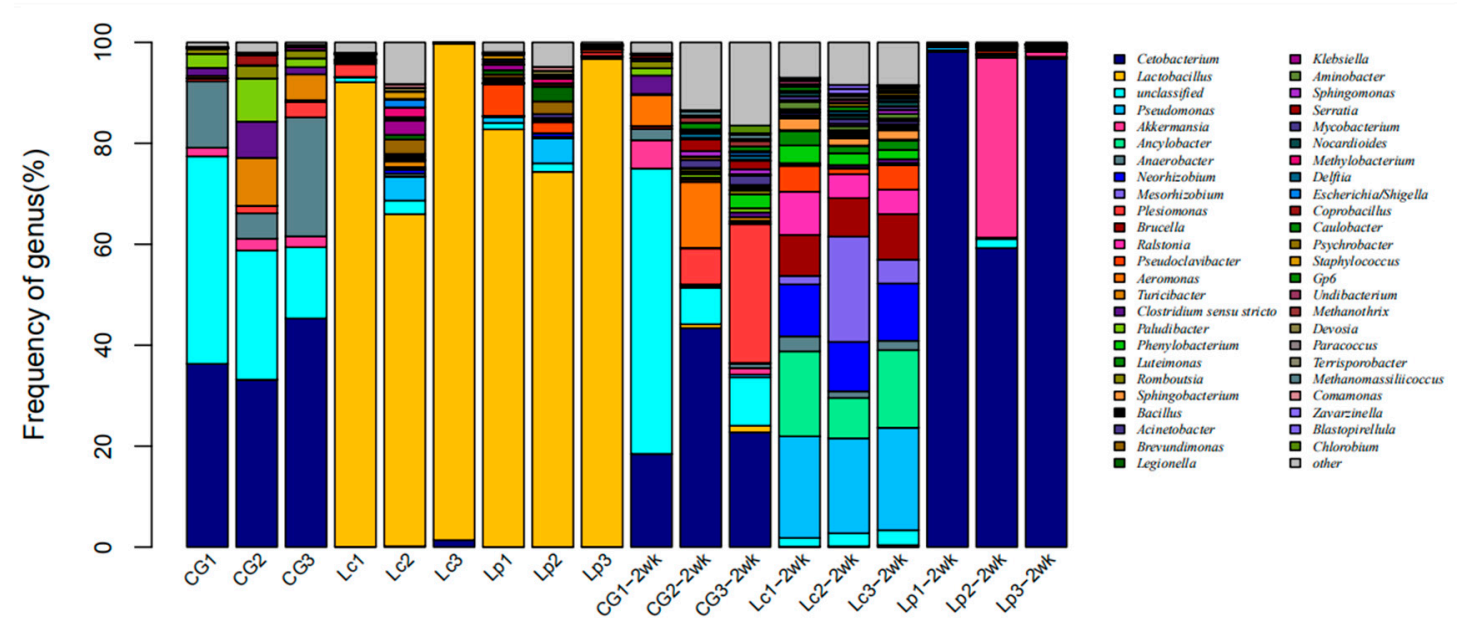

(B)

Figure 5. Gut bacterial community structure at phylum (A) and genus (B) levels. Each treatment has three replicates.

Similarly, at the genus level (Figure 5B), the intestinal flora in CG changed slightly between two time points, while they altered significantly in Lc and Lp groups, e.g., Lactobacillus as expected was the most abundant (accounting for $>80 \%$ of $16 \mathrm{~S}$ reads) genus in both Lc and Lp groups, however, this genus almost disappeared $(<1 \%)$ after two weeks post-cessation, suggesting that Lactobacillus is a transient bacteria strain. Intriguingly, the intestinal bacteria in both group had different changes, the expression of multiple other genera increased in Lc-2wk, such as, Pseudomonas (19.75\%), Ancylobacter (13.41\%), Neorhizobium (10.47\%), Mesorhizobium (9.10\%), Brucella (8.26\%), Ralstonia (6.06\%), Pseudoclavibacter (3.69\%), Phenylobacterium (2.56\%), Luteimonas (2.06\%) and Anaerobacter (2.05\%). However, the predominant genera in Lp-2wk were Cetobacterium $(84.72 \%)$ and Akkermansia $(12.23 \%)$.

\subsection{Functional Analysis of the Intestinal Microflora}

To further analyze the effect of probiotics on gut bacteria, we performed functional analysis based on the community composition of the gut microbiome after probiotics-fed via PICRUSt. KEGG orthologs were classified to level 3. Most of the predicted functional pathways belonged to six main 
categories, i.e., metabolism, organismal systems, genetic information processing, cellular processes, environmental information processing and human diseases (Figure 6). Although there were no significant different abundances among Lc, Lp and CG in metabolism ( $p>0.05)$, five KEGG pathways (Lipid Metabolism, Metabolism of Other Amino Acids, Metabolism of Terpenoids and Polyketides, Xenobiotics Biodegradation and Metabolism and Nucleotide Metabolism) in level 2 and 20 KEGG pathways in level 3 were significantly enriched in Lc $(p<0.05)$. One KEGG pathway (Metabolism of Terpenoids and Polyketides) in level 2 and 10 KEGG pathways in level 3 were significantly enriched in Lp $(p<0.05)$ (Table S2).

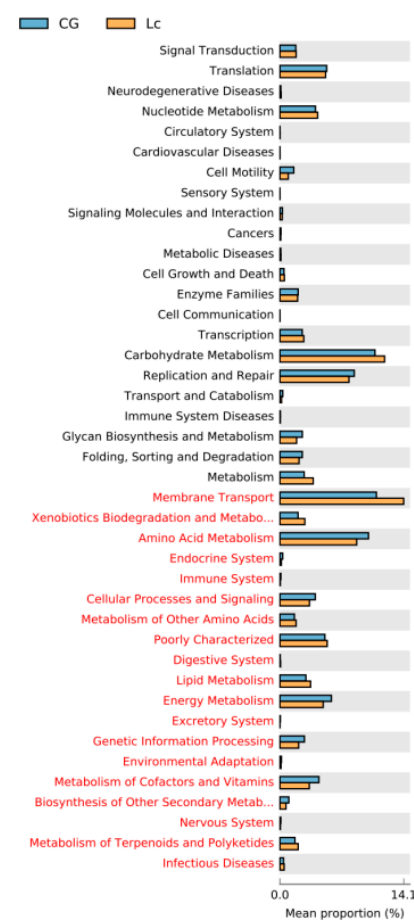

(A)

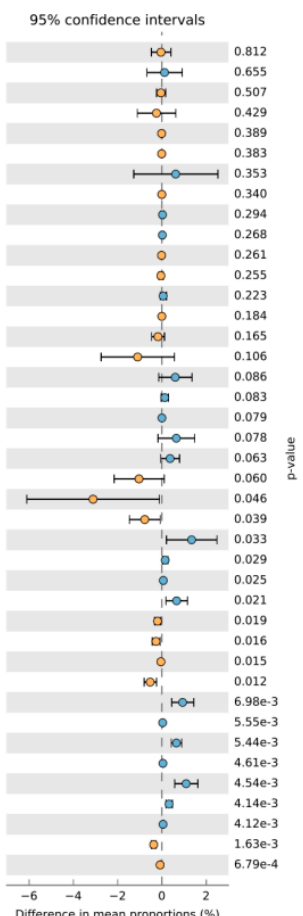

(1)

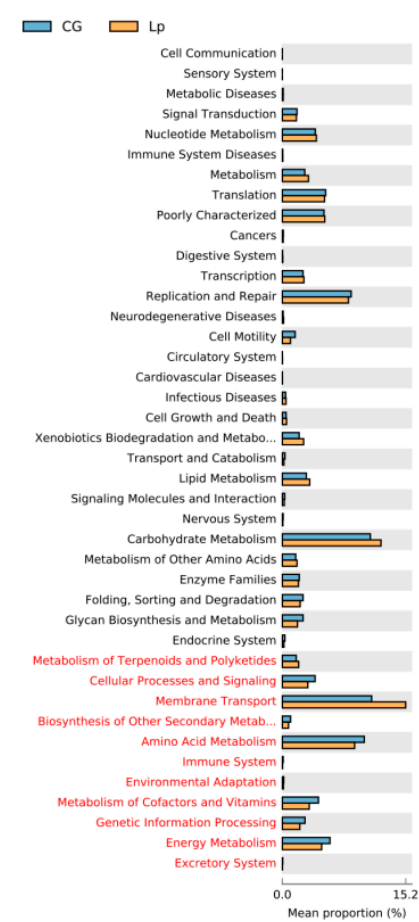

(B)

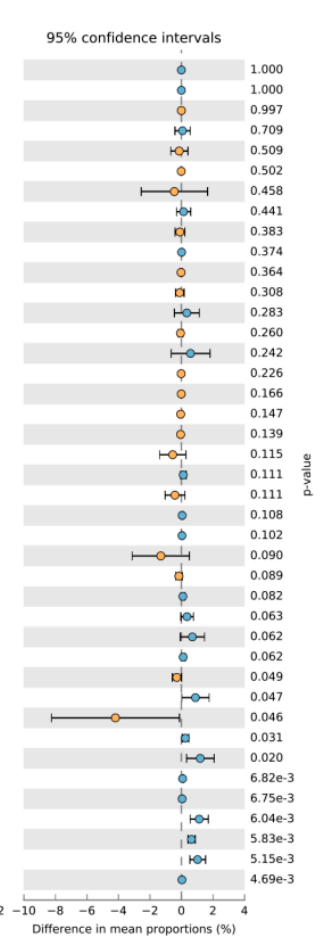

Figure 6. Comparison of normalized abundance data of KEGG pathways of intestinal microbiome from CG group with Lc group (A) and with Lp group (B).

\section{Discussion}

$\mathrm{LAB}$ as probiotics have been widely used as dietary supplements for promoting growth performance and disease resistance of aquatic animals including tilapia [35], common carp [36], sea bass [18], rainbow trout [37], red sea bream [38], and olive flounder [39]. However, few probiotics have been used for channel catfish. Previously, we isolated two new LAB strains, L. casei YYL3 and $L$. plantarum YYL5, with potential antibacterial activity against E. ictaluri, A. hydrophila, and Y. ruckeri, which are common bacterial pathogens of catfishes. In the present study, we found significant increase in growth parameters (WG and SGR) and decrease in FCR in catfish fed with Lc, at $10^{8} \mathrm{cfu} / \mathrm{g}$ level for 4 weeks compared with CG. This is in congruence with the effects of other L. casei strains on aquatic animals. For instances, Lamari et al. [18] reported that L. casei X2 could promote the growth of sea bass larvae. Andani et al. [40] found that commercial feed containing $5 \times 10^{7} \mathrm{cfu} / \mathrm{g}$ of $\mathrm{L}$. casei could improve growth parameters of rainbow trout. This growth enhancement by Lc is probably due to its effect on intestinal microbiota which has been demonstrated to play important roles in digestion, the production of essential vitamins, and protection of the gastrointestinal tract from pathogen colonization [41]. In accordance with this assumption, we found that Lc-feed changed the relative abundance of many genes enriched in metabolism pathways including lipid metabolism, metabolism of other amino acids, metabolism of terpenoids and polyketides, xenobiotics biodegradation and 
metabolism, nucleotide metabolism and carbohydrate metabolism, which could potentially improve feed utilization and growth.

Stimulation of immune system and increase of disease resistance are another important benefits of probiotics [42]. Lysozyme plays an important role in disease defense by its antibacterial activity [43], thus its activity has been frequently used as an indicator of non-specific immune functions, which is of primary importance in combating infections in fish. In this study, Lc supplementation significantly upregulated lysozyme activity in the blood of catfish, which is coincide with higher RPS in Lc following challenge with E. ictaluri. This indicates that dietary supplementation of Lc can improve disease resistance in catfish in comparison with control and Lp. Similarly, significant stimulation of fish immunity by dietary Lc has been reported in previous studies on zebrafish [44], and rainbow trout [40].

Although the positive effects of L. plantarum in aquaculture animals have been investigated including enhancement of the growth performance [11,14,25], and immune response $[15,35,45]$ and inhibition of adhesion of pathogenic microorganisms $[9,10]$. In this study, we didn't find any significant effect of Lp on growth performance, immune parameters and RPS of catfish compared to CG. Similar to our study, Lee et al. [46] revealed that oral administration of diet with different levels of L. plantarum KCTC3928 $\left(10^{6} \sim 10^{8} \mathrm{cfu} / \mathrm{g}\right)$ could not improve growth performance of Japanese eel in terms of weight gain, feed efficiency ratio, and protein efficiency ratio. Beck et al. [45] found that the single L. plantarum FGL0001-fed group of olive flounder did not produce a statistical increase in weight gain compared to the control. Butprom et al. [47] found that L. plantarum C014 could promote lysozyme activity in hybrid catfish after 45 days of feeding but not 30 days. The observed different effects of L. plantarum on growth performance and immune response of different aquatic animals may be attributed to the host species-specific response, and different active ingredients secreted by different L. plantarum strains.

There are several studies on the composition of intestinal microbiota in channel catfish. Larsen et al. [48] investigated the gut microbiota of channel catfish, Micropterus salmoides, and Lepomis macrochirus from the same pond using 16S rRNA pyrosequencing, and found that microbiota differed significantly between fish species in terms of bacterial species evenness. However, all gut communities shared the dominant species Cetobacterium somerae, Plesiomonas shigelloides and F. mortiferum which belong to the phylum Fusobacteria. Bledsoe et al. [49] surveyed the intestinal microbiota of channel catfish with high-throughput DNA sequencing of 16S rRNA V4 gene amplicons derived from fish at different ages, and revealed that microbial communities inhabiting the intestines of catfish early in life were dynamic, with sharp transition occurring up to 125 days post hatch (dph) when the microbiota somewhat stabilized. The most abundant microbiota in the catfish after $3 \mathrm{dph}$ are Bacteroidetes, Firmicutes, Fusobacteria, and Proteobacteria, with the species C.somerae and P. shigelloides. In agreement with these findings, our study showed that the Fusobacteria, Bacteroidetes, Firmicutes and Proteobacteria were the dominant phyla, with the genus Cetobacterium showing the highest abundance in the control group (120dph-150dph).

After 4 weeks feeding trail, Lactobacillus reduced the alpha diversity of the intestinal microbiota. At the genus level, Lactobacillus were the dominant bacteria of Lc and Lp groups, and the proportion of Lactobacillus were $85.39 \%$ and $84.58 \%$, respectively. Pseudomonas was the second and third most dominant microbiota of Lc and Lp groups, separately. After 2 weeks cessation of probiotic administration, the proportion of Pseudomonas increased from $1.59 \%$ in Lc to $19.75 \%$ in Lc-2wk, becoming the first dominant bacteria, while this bacterium was not detected in CG, Lc, Lp and CG-2wk, and only a very low level in Lp-2wk. Because this bacterium that is widely distributed in the fish and shrimp, can produce lipase, increase lipid utilization rate and improve growth performance [50-52], it has been regarded as candidate probiotics in grass carp and is an important biological control agent in aquaculture [53]. Aeromonas are thought to be opportunistic pathogens in freshwater fish, which exist in the breeding environment and intestinal tract. Bacterial diseases occur easily when the immunity of fish declined, or the environment changed. In our study, the relative abundance of Aeromonas in the intestinal of CG increased from $0.13 \%$ to $6.43 \%$ in CG-2wk. It is reported that fish digestive tract is a reservoir for many opportunistic pathogens [54,55], whereas both Lc and Lp exhibited significant 
inhibition of Aeromonas, suggesting that the Lc and Lp dramatically changed the composition of intestinal microbiota of channel catfish.

\section{Conclusions}

This study evaluated two probiotics Lc YYL3 and Lp YYL5 in channel catfish aquaculture. Dietary supplementation with Lc YYL3 improved the growth performance and disease resistance in channel catfish. Dietary supplementation with Lc YYL3 or Lp YYL5 reduced the alpha diversity and dramatically changed the composition of the intestinal microbiota in channel catfish.

Supplementary Materials: The following are available online at http://www.mdpi.com/2076-2615/9/12/1005/s1. Figure S1: Heatmap showing the gut microbial compositions of channel catfish right after feeding with basal diet and Lactobacillus-containing diets for 4 weeks and after 2 more weeks cessation. Table S1: The first 10 genera in the 6 groups at genus level. Table S2: Mean relative abundances (\%) of selected KEGG pathways related to metabolism.

Author Contributions: Conceptualization, H.Z., X.Y., and L.X., methodology, H.Z., and H.W., formal analysis, H.Z. and H.W., investigation, H.W. and L.J., writing-original draft preparation, H.Z.; Writing-review and editing, H.W., K.H. and M.Z.

Funding: This study was funded by Central Public-interest Scientific Institution Basal Research Fund, CAFS (NO. 2019A006).

Acknowledgments: The authors thank Zhou who provided Edwardsiella ictaluri using for the challenge test.

Conflicts of Interest: The authors declare no conflict of interest.

\section{References}

1. Burgos, F.A.; Ray, C.L.; Arias, C.R. Bacterial diversity and community structure of the intestinal microbiome of Channel Catfish (Ictalurus punctatus) during ontogenesis. Syst. Appl. Microbiol. 2018, 41, 494-505. [CrossRef] [PubMed]

2. China, B.O.F.I. Chinese Fishery Statistical Yearbook; China Agriculture Press: Beijing, China, 2018.

3. Wang, R.H.; Xiao, T.Y.; Zeng, L.B.; Liu, X.Y.; Zhou, Y.; Ma, J. Generation and use of Edwardsiella ictaluri ghosts as a vaccine against enteric septicemia of catfish (ESC). Aquaculture 2016, 456, 9-15. [CrossRef]

4. Peatman, E.; Mohammed, H.; Kirby, A.; Shoemaker, C.A.; Yildirim-Aksoy, M.; Beck, B.H. Mechanisms of pathogen virulence and host susceptibility in virulent Aeromonas hydrophila infections of channel catfish (Ictalurus punctatus). Aquaculture 2018, 482, 1-8. [CrossRef]

5. Dawood, M.A.O.; Koshio, S. Recent advances in the role of probiotics and prebiotics in carp aquaculture: A review. Aquaculture 2016, 454, 243-251. [CrossRef]

6. Zorriehzahra, M.J.; Delshad, S.T.; Adel, M.; Tiwari, R.; Karthik, K.; Dhama, K.; Lazado, C.C. Probiotics as beneficial microbes in aquaculture: An update on their multiple modes of action: A review. Vet. Q. 2016, 36, 228-241. [CrossRef]

7. Bernardeau, M.; Guguen, M.; Vernoux, J.P. Beneficial lactobacilli in food and feed: Long-term use, biodiversity and proposals for specific and realistic safety assessments. FEMS Microbiol. Rev. 2006, 30, 487-513. [CrossRef]

8. Ringø, E.; Gatesoupe, F. Lactic acid bacteria in fish: A review. Aquaculture 1998, 160, 177-203. [CrossRef]

9. Ren, P.F.; Xu, L.; Yang, Y.L.; He, S.X.; Liu, W.S.; Ringø, E.; Zhou, Z.G. Lactobacillus planarum subsp. plantarum JCM 1149 vs. Aeromonas hydrophila NJ-1 in the anterior intestine and posterior intestine of hybrid tilapia Oreochromis niloticus $+\times$ Oreochromis aureuso': An ex vivo study. Fish Shellfish Immun. 2013, 35, 146-153.

10. Gao, Q.X.; Gao, Q.; Min, M.H.; Zhang, C.J.; Peng, S.M.; Shi, Z.H. Ability of Lactobacillus plantarum lipoteichoic acid to inhibit Vibrio anguillarum-induced inflammation and apoptosis in silvery pomfret (Pampus argenteus) intestinal epithelial cells. Fish Shellfish Immun. 2016, 54, 573-579. [CrossRef]

11. Yu, L.L.; Zhai, Q.X.; Zhu, J.M.; Zhang, C.C.; Li, T.Q.; Liu, X.M.; Zhao, J.X.; Zhang, H.; Tian, F.W.; Chen, W. Dietary Lactobacillus plantarum supplementation enhances growth performance and alleviates aluminum toxicity in tilapia. Ecotoxicol. Environ.Saf. 2017, 143, 307-314. [CrossRef]

12. Zheng, X.T.; Duan, Y.F.; Dong, H.B.; Zhang, J.S. Effects of Dietary Lactobacillus plantarum on Growth Performance, Digestive Enzymes and Gut Morphology of Litopenaeus vannamei. Probiotics Antimicro 2018, 10, 504-510. [CrossRef] [PubMed] 
13. Dawood, M.A.O.; Koshio, S.; Ishikawa, M.; Yokoyama, S. Effects of heat killed Lactobacillus plantarum (LP20) supplemental diets on growth performance, stress resistance and immune response of red sea bream, Pagrus major. Aquaculture 2015, 442, 29-36. [CrossRef]

14. Son, V.M.; Chang, C.C.; Wu, M.C.; Guu, Y.K.; Chiu, C.H.; Cheng, W.T. Dietary administration of the probiotic, Lactobacillus plantarum, enhanced the growth, innate immune responses, and disease resistance of the grouper Epinephelus coioides. Fish Shellfish Immun. 2009, 26, 691-698. [CrossRef] [PubMed]

15. Beck, B.R.; Song, J.H.; Park, B.S.; Kim, D.; Kwak, J.H.; Do, H.K.; Kim, A.R.; Kim, W.J.; Song, S.K. Distinct immune tones are established by Lactococcus lactis BFE920 and Lactobacillus plantarum FGL0001 in the gut of olive flounder (Paralichthys olivaceus). Fish Shellfish Immun. 2016, 55, 434-443. [CrossRef] [PubMed]

16. Giri, S.S.; Sukumaran, V.; Oviya, M. Potential probiotic Lactobacillus plantarum VSG3 improves the growth, immunity, and disease resistance of tropical freshwater fish, Labeorohita. Fish Shellfish Immun. 2013, 34, 660-666. [CrossRef] [PubMed]

17. Aktas, B.; Wolfe, T.J.D.; Safdar, N.; Darien, B.J.J.; Steele, J.L. The Impact of Lactobacillus casei on the Composition of the Cecal Microbiota and Innate Immune System Is Strain Specific. PLoS ONE 2016, 11, e156374. [CrossRef]

18. Lamari, F.; Castex, M.; Larcher, T.; Ledevin, M.; Mazurais, D.; Bakhrouf, A.; Gatesoupe, F.J. Comparison of the effects of the dietary addition of two lactic acid bacteria on the development and conformation of sea bass larvae, Dicentrarchus labrax, and the influence on associated microbiota. Aquaculture 2013, 376-379, 137-145. [CrossRef]

19. Abasali, H.; Mohamad, S. Effect of Dietary Supplementation with Probiotic on Reproductive Performance of Female Livebearing Ornamental Fish. Res. J. Anim. Sci. 2010, 4, 103-107.

20. Zhang, D.D.; Beck, B.H.; Lange, M.D.; Zhao, H.G.; Thongda, W.; Ye, Z.; Li, C.; Peatman, E. Impact of oral and waterborne administration of rhamnolipids on the susceptibility of channel catfish (Ictalurus punctatus) to Flavobacterium columnare infection. Fish Shellfish Immun. 2017, 60, 44-49. [CrossRef]

21. Li, E.C.; Lim, C.; Klesius, P. Enhancement Effects of Dietary Wheat Distiller's Dried Grains with Solubles on Growth, Immunity, and Resistance to Edwardsiella ictaluri Challenge of Channel Catfish, Ictalurus punctatus. J. WorldAquac. Soc. 2012, 43, 814-827.

22. Liu, C.H.; Wu, K.C.; Chu, T.W.; Wu, T.M. Dietary supplementation of probiotic, Bacillus subtilis E20, enhances the growth performance and disease resistance against Vibrio alginolyticus in parrot fish (Oplegnathus fasciatus). Aquacult. Int. 2018, 26, 63-74. [CrossRef]

23. Xia, Y.; Lu, M.X.; Chen, G.; Cao, J.M.; Gao, F.Y.; Wang, M.; Liu, Z.G.; Zhang, D.F.; Zhu, H.P.; Yi, M.M. Effects of dietary Lactobacillus rhamnosus JCM1136 and Lactococcus lactis subsp. lactis JCM5805 on the growth, intestinal microbiota, morphology, immune response and disease resistance of juvenile Nile tilapia, Oreochromis niloticus. Fish Shellfish Immun. 2018, 76, 368-379.

24. Gupta, A.; Sehgal, H.S.; Sehgal, G.K. Growth and carcass composition of giant freshwater prawn, Macrobrachium rosenbergii (De Man), fed different isonitrogenous and isocaloric diets. Aquac. Res. 2007, 38, 1355-1363. [CrossRef]

25. Doan, H.V.; Hoseinifar, S.H.; Tapingkae, W.; Tongsiri, S.; Khamtavee, P. Combined administration of low molecular weight sodium alginate boosted immunomodulatory, disease resistance and growth enhancing effects of Lactobacillus plantarum in Nile tilapia (Oreochromis niloticus). Fish Shellfish Immun. 2016, 58, 678-685. [CrossRef] [PubMed]

26. Doan, H.V.; Doolgindachbaporn, S.; Suksri, A. Effects of low molecular weight agar and Lactobacillus plantarum on growth performance, immunity, and disease resistance of basa fish (Pangasius bocourti, Sauvage 1880). Fish Shellfish Immun. 2014, 41, 340-345. [CrossRef]

27. Liu, H.T.; Wang, S.F.; Cai, Y.; Guo, X.H.; Cao, Z.J.; Zhang, Y.Z.; Liu, S.B.; Yuan, W.; Zhu, W.W.; Zheng, Y.; et al. Dietary administration of Bacillus subtilis HAINUP40 enhances growth, digestive enzyme activities, innate immune responses and disease resistance of tilapia, Oreochromis niloticus. Fish Shellfish Immun. 2017, 60, 326-333. [CrossRef]

28. Huang, W.; Cheng, Z.Q.; Lei, S.N.; Liu, L.Y.; Lv, X.; Chen, L.H.; Wu, M.H.; Wang, C.; Tian, B.Y.; Song, Y.K. Community composition, diversity, and metabolism of intestinal microbiota in cultivated European eel (Anguilla anguilla). Appl. Microbiol.Biot. 2018, 102, 4143-4157. [CrossRef]

29. Zhang, J.J.; Kobert, K.; Flouri, T.; Stamatakis, A. PEAR: A fast and accurate Illumina Paired-End reAdmergeR. Bioinformatics 2014, 30, 614-620. [CrossRef] 
30. Schmieder, R.; Edwards, R. Quality control and preprocessing of metagenomic datasets. Bioinformatics 2011, 27, 863-864. [CrossRef]

31. Edgar, R.C. Search and clustering orders of magnitude faster than BLAST. Bioinformatics 2010, 26, $2460-2461$. [CrossRef]

32. Schloss, P.D.; Westcott, S.L.; Ryabin, T.; Hall, J.R.; Hartmann, M.; Hollister, E.B.; Lesniewski, R.A.; Oakley, B.B.; Parks, D.H.; Robinson, C.J.; et al. Introducing mothur: Open-Source, Platform-Independent, Community-Supported Software for Describing and Comparing Microbial Communities. Appl. Environ. Microb. 2009, 75, 7537-7541. [CrossRef] [PubMed]

33. Caporaso, J.G.; Kuczynski, J.; Stombaugh, J.; Bittinger, K.; Bushman, F.D.; Costello, E.K.; Fierer, N.; Pena, A.G.; Goodrich, J.K.; Gordon, J.I.; et al. QIIME allows analysis of high-throughput community sequencing data. Nat. Methods 2010, 7, 335-336. [CrossRef] [PubMed]

34. Langille, M.G.I.; Zaneveld, J.; Caporaso, J.G.; McDonald, D.; Knights, D.; Reyes, J.A.; Clemente, J.C.; Burkepile, D.E.; Thurber, R.L.V.; Knight, R.; et al. Predictive functional profiling of microbial communities using $16 \mathrm{~S}$ rRNA marker gene sequences. Nat. Biotechnol. 2013, 31, 814-821. [CrossRef] [PubMed]

35. Doan, H.V.; Hoseinifar, S.H.; Dawood, M.A.O.; Chitmanat, C.; Tayyamath, K. Effects of Cordyceps militaris spent mushroom substrate and Lactobacillus plantarum on mucosal, serum immunology and growth performance of Nile tilapia (Oreochromis niloticus). Fish Shellfish Immun. 2017, 70, 87-94. [CrossRef]

36. Soltani, M.; Abdy, E.; Alishahi, M.; Mirghaed, A.T.; Hosseini-Shekarabi, P. Growth performance, immune-physiological variables and disease resistance of common carp (Cyprinus carpio) orally subjected to different concentrations of Lactobacillus plantarum. Aquac. Int. 2017, 25, 1913-1933. [CrossRef]

37. Vendrell, D.; Balcázar, J.L.; Blas, I.D.; Ruiz-Zarzuela, I.; Gironés, O.; Múzquiz, J.L. Protection of rainbow trout (Oncorhynchus mykiss) from lactococcosis by probiotic bacteria. Comp. Immun. Microbiol. Infect. Dis. 2008, 31, 337-345. [CrossRef]

38. Dawood, M.A.O.; Koshio, S.; Ishikawa, M.; Yokoyama, S.; Basuini, M.F.E.; Hossain, M.S.; Nhu, T.H.; Dossou, S.; Moss, A.S. Effects of dietary supplementation of Lactobacillus rhamnosus or/and Lactococcus lactis on the growth, gut microbiota and immune responses of red sea bream, Pagrus major. Fish Shellfish Immun. 2016, 49, 275-285. [CrossRef]

39. Kim, D.; Beck, B.R.; Heo, S.; Kim, J.J.; Kim, H.D.; Lee, S.; Kim, Y.; Oh, S.Y.; Lee, K.; Do, H.K.; et al. Lactococcus lactis BFE920 activates the innate immune system of olive flounder (Paralichthys olivaceus), resulting in protection against Streptococcus iniae infection and enhancing feed efficiency and weight gain in large-scale field studies. Fish Shellfish Immun. 2013, 35, 1585-1590. [CrossRef]

40. Andani, H.R.R.; Tukmechi, A.; Meshkini, S.; Sheikhzadeh, N. Antagonistic activity of two potential probiotic bacteria from fish intestines and investigation of their effects on growth performance and immune response in rainbow trout (Oncorhynchus mykiss). J. Appl. Ichthyol. 2012, 28, 728-734. [CrossRef]

41. O'Hara, A.M.; Shanahan, F. The gut flora as a forgotten organ. EMBO Rep. 2006, 7, 688-693. [CrossRef]

42. Aly, S.M.; Ahmed, Y.A.; Ghareeb, A.A.; Mohamed, M.F. Studies on Bacillus subtilis and Lactobacillus acidophilus, as potential probiotics, on the immune response and resistance of Tilapia nilotica (Oreochromis niloticus) to challenge infections. Fish Shellfish Immun. 2008, 25, 128-136. [CrossRef] [PubMed]

43. Choi, S.H.; Park, K.H.; Yoon, T.J.; Kim, J.B.; Jang, Y.S.; Choe, C.H. Dietary Korean mistletoe enhances cellular non-specific immune responses and survival of Japanese eel (Anguilla japonica). Fish Shellfish Immun. 2008, 24, 67-73. [CrossRef] [PubMed]

44. Qin, C.B.; Zhang, Z.; Wang, Y.B.; Li, S.N.; Ran, C.; Hu, J.; Xie, Y.D.; Li, W.F.; Zhou, Z.G. EPSP of L. casei BL23 Protected against the Infection Caused by Aeromonas veronii via Enhancement of Immune Response in Zebrafish. Front. Microbiol. 2017, 8, 2406. [CrossRef] [PubMed]

45. Beck, B.R.; Kim, D.; Jeon, J.; Lee, S.M.; Kim, H.K.; Kim, O.J.; Lee, J.I.; Suh, B.S.; Do, H.K.; Lee, K.H.; et al. The effects of combined dietary probiotics Lactococcus lactis BFE920 and Lactobacillus plantarum FGL0001 on innate immunity and disease resistance in olive flounder (Paralichthys olivaceus). Fish Shellfish Immun. 2015, 42, 177-183. [CrossRef] [PubMed]

46. Lee, S.; Katya, K.; Park, Y.J.; Won, S.H.; Seong, M.J.; Hamidoghli, A.; Bai, S.C. Comparative evaluation of dietary probiotics Bacillus subtilis WB60 and Lactobacillus plantarum KCTC3928 on the growth performance, immunological parameters, gut morphology and disease resistance in Japanese eel, Anguilla japonica. Fish Shellfish Immun. 2017, 61, 201-210. [CrossRef] 
47. Butprom, S.; Phumkhachorn, P.; Rattanachaikunsopon, P. Effect of Lactobacillus plantarum C014 on Innate Immune Response and Disease Resistance against Aeromonas hydrophila in Hybrid Catfish. Sci. World J. 2013, 2013, 1-6. [CrossRef]

48. Larsen, A.M.; Mohammed, H.H.; Arias, C.R. Characterization of the gut microbiota of three commercially valuable warmwater fish species. J. Appl. Microbiol. 2014, 116, 1396-1404. [CrossRef]

49. Bledsoe, J.W.; Peterson, B.C.; Swanson, K.S.; Small, B.C. Ontogenetic Characterization of the Intestinal Microbiota of Channel Catfish through 16S rRNA Gene Sequencing Reveals Insights on Temporal Shifts and the Influence of Environmental Microbes. PLoS ONE 2016, 11, e166379. [CrossRef]

50. Tanasomwang, V.; Muroga, K. Intestinal microflora of rockfish Sebastes schlegeli, tiger puffer Takifugu rubripes and red grouper Epinephelus akaara at their larval and juvenile stages. Nippon Suisan Gakk. 1989, 55, 1371-1377. [CrossRef]

51. Xun, P.W.; Lin, H.Z.; Wang, R.X.; Huang, Z.; Zhou, C.P.; Yu, W.; Huang, Q.Q.; Tan, L.J.; Wang, Y.; Wang, J. Effects of dietary vitamin $B_{1}$ on growth performance, intestinal digestion and absorption, intestinal microflora and immune response of juvenile golden pompano (Trachinotus ovatus). Aquaculture 2019, 506, 75-83. [CrossRef]

52. Mouchet, M.A.; Bouvier, C.; Bouvier, T.; Troussellier, M.; Escalas, A.; Mouillot, D. Genetic difference but functional similarity among fish gut bacterial communities through molecular and biochemical fingerprints. FEMS Microbiol. Ecol. 2012, 79, 568-580. [CrossRef] [PubMed]

53. Wu, S.G.; Wang, G.T.; Angert, E.R.; Wang, W.W.; Li, W.X.; Zou, H. Composition, diversity, and origin of the bacterial community in grass carp intestine. PLoS ONE 2012, 7, 1-11. [CrossRef] [PubMed]

54. Pond, M.J.; Stone, D.M.; Alderman, D.J. Comparison of conventional and molecular techniques to investigate the intestinal microflora of rainbow trout (Oncorhynchus mykiss). Aquaculture 2006, 261, 194-203. [CrossRef]

55. Wu, S.; Gao, T.H.; Zheng, Y.Z.; Wang, W.W.; Cheng, Y.Y.; Wang, G.T. Microbial diversity of intestinal contents and mucus in yellow catfish (Pelteobagrus fulvidraco). Aquaculture 2010, 303, 1-7. [CrossRef]

(C) 2019 by the authors. Licensee MDPI, Basel, Switzerland. This article is an open access article distributed under the terms and conditions of the Creative Commons Attribution (CC BY) license (http://creativecommons.org/licenses/by/4.0/). 